\title{
UNIFORM ASYMPTOTIC STABILITY VIA LIAPUNOV-RAZUMIKHIN TECHNIQUE
}

\author{
JAMES H. LIU
}

(Communicated by Hal. L. Smith)

\begin{abstract}
The Liapunov-Razumikhin technique is applied to obtain the uniform asymptotic stability for linear integrodifferential equations in Hilbert spaces,
\end{abstract}

$$
x^{\prime}(t)=A\left[x(t)+\int_{\#}^{t} F(t-s) x(s) d s\right], \quad t \geq t_{0} \geq 0(\#=0 \text { or }-\infty),
$$

which occur in viscoelasticity and in heat conduction for materials with memory.

\section{INTRODUCTION}

In order to introduce the equations that we will study, let us consider the following heat equation for material with memory (see, e.g., [4]):

$$
\left\{\begin{array}{l}
q(t, x)=-E u_{x}(t, x)-\int_{\#}^{t} b(t-s) u_{x}(s, x) d s \quad(\#=0 \text { or }-\infty), \\
u_{t}(t, x)=-\partial q(t, x) / \partial x+f(t, x) .
\end{array}\right.
$$

The first equation gives the heat flux, and the second is the balance equation. Equation (1.1) can be written as (assuming $E=1$ )

$$
u_{t}(t, x)=\frac{\partial^{2}}{\partial x^{2}}\left[u(t, x)+\int_{\#}^{t} b(t-s) u(s, x) d s\right]+f(t, x) .
$$

Thus we see that equations

$$
\begin{aligned}
& x^{\prime}(t)=A\left[x(t)+\int_{0}^{t} F(t-s) x(s) d s\right], \\
& \quad t \geq t_{0} \geq 0, x(s)=\phi(s), 0 \leq s \leq t_{0},
\end{aligned}
$$

and

$$
x^{\prime}(t)=A\left[x(t)+\int_{-\infty}^{t} F(t-s) x(s) d s\right], \quad t \geq t_{0} \geq 0, x(s)=\phi(s), s \leq t_{0},
$$

Received by the editors December 2, 1992 and, in revised form, December 10, 1993.

1991 Mathematics Subject Classification. Primary 45K05, 34G10.

Key words and phrases. Uniform asymptotic stability, Liapunov-Razumikhin technique. 
can arise naturally in applications. See Grimmer and Liu [3] for other comments and another example in viscoelasticity. Here, operator $A$ generates a strongly continuous semigroup and $F(t)$ is a bounded operator for $t \geq 0$ on a real Hilbert space $(X,\|\cdot\|)$.

We also note that equations of this type, where operator $A$ applies to the addition of $x(t)$ and an integral term, have received some attention recently. For example, in Fabiano and Ito [2], the equation of a linear viscoelastic beam is formulated as

$$
x^{\prime \prime}(t)+A\left[E x(t)-\int_{-r}^{t} g(s) x(s+t) d s\right]=f(t),
$$

on a Hilbert space with $A$ a positive definite selfadjoint unbounded operator. Well-posedness and convergence of approximation schemes are studied under certain assumptions on $g$.

We will study uniform asymptotic stability for Eqs. (1.3)-(1.4) in this paper. First, we give definitions. (Note that $x \equiv 0$ satisfies Eq. (1.3) and Eq. (1.4).)

Definition 1.1. For any $t_{0} \geq 0$ and any continuous function $\phi$ on $\left[0, t_{0}\right]$, a solution of Eq. (1.3) is a function $x:[0, \infty) \rightarrow X$ satisfying Eq. (1.3) for $t \geq t_{0}$ and $x(s)=\phi(s)$ for $s \in\left[0, t_{0}\right]$. The solution of Eq. (1.4) is defined accordingly.

Definition 1.2. The solution $x \equiv 0$ of Eq. (1.3) is stable if given $\varepsilon>0$ and $t_{0} \geq 0$, there exists a $\delta=\delta\left(\varepsilon, t_{0}\right)>0$ such that $\left[\|\phi(s)\|<\delta\right.$ on $\left[0, t_{0}\right]$ and $x\left(t, t_{0}, \phi\right)$ being a solution of Eq. (1.3)] imply $\left\|x\left(t, t_{0}, \phi\right)\right\|<\varepsilon$ for $t \geq t_{0}$. It is uniformly stable if it is stable and the $\delta$ is independent of $t_{0}$. For Eq. (1.4), we define them accordingly.

Definition 1.3. The solution $x \equiv 0$ of Eq. (1.3) is uniformly asymptotically stable if it is uniformly stable and there exists a constant $r>0$ and for any $\varepsilon>0$ there exists $T=T(\varepsilon)>0$ such that $\left[\|\phi(s)\|<r\right.$ on $\left[0, t_{0}\right]$ and $x\left(t, t_{0}, \phi\right)$ being a solution of Eq. (1.3)] imply $\left\|x\left(t, t_{0}, \phi\right)\right\|<\varepsilon$ for $t \geq T+t_{0}$. For Eq. (1.4), we define them accordingly.

Remark. In these definitions, it is implied that $x(t)+\int_{\#}^{t} F(t-s) x(s) d s \in D(A)$ for $t \geq t_{0}$, where \#=0 or $-\infty$ and $D$ means domain.

Note that in many applications, the important case is the "initial value problem" when $t_{0}=0$. For example, in Seifert [6,7], where the initial value problem was studied, the case for $t_{0}=0$ was also defined as "stable" and "asymptotically stable" (i.e., treat $t_{0}=0$ in Definitions 1.1-1.3).

In [3], where initial value problems were concerned, we used the definitions of Seifert [6,7], and studied the existence and uniqueness of solutions, as well as boundedness and stability for Eqs. (1.3)-(1.4) (where $t_{0}=0$ ).

However, as remarked in Burton $[1$, p. 70$]$, for $\phi$ given on $\left[0, t_{0}\right]$, one can translate the problem into an initial value problem (begins at $x(0)=\phi\left(t_{0}\right)$ or $\left.x(s)=\phi\left(t_{0}+s\right), s \leq 0\right)$. So results in [3] concerning existence and uniqueness are still valid if Definition 1.1 (here) is used. Also, one can check that the conditions in [3] imply uniform stability if Definitions 1.1-1.2 (here) are used. Because, for example, in the proof of Theorem 1 of Seifert [6], which is used in [3], we only need to change $V\left(0, x_{0}\right)$ on page 426 (line 6) to $\max _{s \leq t_{0}} V(s, \phi(s))$, then all other proofs can go through. 
Therefore, we remark that the existence, uniqueness, and uniform stability for Eqs. (1.3)-(1.4) (according to Definitions 1.1-1.2 here) are known. And we will continue to study uniform asymptotic stability of Eqs. (1.3)-(1.4) in this paper.

Here, we also proceed as in [3] and let

$$
w(t)=x(t)+\int_{0}^{t} F(t-s) x(s) d s, \quad t \geq t_{0},
$$

and rewrite Eq. (1.3) as

$$
\begin{aligned}
{\left[\begin{array}{c}
x(t) \\
w(t)
\end{array}\right]^{\prime}=} & {\left[\begin{array}{cc}
0 & A \\
F(0) & A
\end{array}\right]\left[\begin{array}{c}
x(t) \\
w(t)
\end{array}\right] } \\
& +\int_{0}^{t}\left[\begin{array}{cc}
0 & 0 \\
F^{\prime}(t-s) & 0
\end{array}\right]\left[\begin{array}{c}
x(s) \\
w(s)
\end{array}\right] d s, \quad t \geq t_{0} \geq 0, \\
& (x(s), w(s))=(\phi(s), \psi(s)), \quad 0 \leq s \leq t_{0} .
\end{aligned}
$$

For Eq. (1.4), we let

$$
w(t)=x(t)+\int_{-\infty}^{t} F(t-s) x(s) d s, \quad t \geq t_{0},
$$

and rewrite Eq. (1.4) as

$$
\begin{aligned}
{\left[\begin{array}{c}
x(t) \\
w(t)
\end{array}\right]^{\prime}=} & {\left[\begin{array}{cc}
0 & A \\
F(0) & A
\end{array}\right]\left[\begin{array}{l}
x(t) \\
w(t)
\end{array}\right] } \\
& +\int_{-\infty}^{t}\left[\begin{array}{cc}
0 & 0 \\
F^{\prime}(t-s) & 0
\end{array}\right]\left[\begin{array}{c}
x(s) \\
w(s)
\end{array}\right] d s, \quad t \geq t_{0} \geq 0, \\
& (x(s), w(s))=(\phi(s), \psi(s)), \quad s \leq t_{0} .
\end{aligned}
$$

Thus it is clear that in order to prove the uniform asymptotic stability for Eq. (1.3) or (1.4), we only need to prove that property for Eq. (1.7) or (1.9).

Conditions for a "stable matrix" in finite-dimensional space cases are generalized to operators $F(0)$ and $A$, so that the leading matrix in Eq. (1.7) or (1.9) plays a role of "stable operator". Base on that we can define a Liapunov function and show that it has certain properties.

Due to the nature of Eq. (1.3) or (1.4), we have to make use of some results of Liapunov-Razumikhin type which can handle the integral part in the derivative of the Liapunov function. The following theorem, modified after Theorem 2 of Grimmer and Seifert [5], is very useful here. We omit the proof since it is the same as that in [5]. We let $z(t)=(x(t), w(t))^{T}$ in Eq. (1.7) or (1.9), and let $h$ be a continuous function such that $h(t)>t$ for $t>0$.

Theorem 1.4. Suppose that $V: Z \equiv X \times X \rightarrow R^{+} \equiv[0, \infty)$ satisfies

(i) $a(\|z\|) \leq V(z) \leq b(\|z\|)$ for all $z \in Z$ where $a, b: R^{+} \rightarrow R^{+}$are continuous and increasing with $a(r) \rightarrow \infty$ as $r \rightarrow \infty$. (Here $\|z\|=\|x\|+\|w\|$.)

(ii) Given $M>0$, there exist monotone sequences of positive reals $\left\{r_{j}\right\}$ and $\left\{u_{j}\right\}$ with $r_{j} \rightarrow \infty$ and $u_{j} \rightarrow 0$ as $j \rightarrow \infty$, and continuous functions $w_{j}(s)$ which are positive on the set $u_{j} / 2 \leq s \leq M$ so that given a solution $z(s)=$ $z\left(s, t_{0}, \xi\right)$ of $E q$. (1.7) (or of Eq. (1.9)) with $\|z(s)\| \leq M$ for all $s$, if for some $t \geq r_{j}+t_{0}$ one has $u_{j} \leq\|z(t)\|$ and $V(z(s))<h(V(z(t)))$ for $t-r_{j} \leq s \leq t$, then $V^{\prime}(z(t)) \leq-w_{j}(\|z(t)\|)$. 
Then every bounded solution of Eq. (1.7) (or of Eq. (1.9)) tends to zero as $t \rightarrow$ $\infty$. In particular, given $\varepsilon>0$ and $M>0$, there is $T=T(\varepsilon, M)>0$ so that for a solution $z(\cdot)=z\left(\cdot, t_{0}, \xi\right)$ of $E q$. (1.7) (or of Eq. (1.9)) with $\|z(t)\| \leq M$ for all $t$, then $\|z(t)\|<\varepsilon$ for $t \geq T+t_{0}$.

In the next section we will apply this result to Eq. (1.7) and Eq. (1.9) and obtain the uniform asymptotic stability.

\section{THE ASYMPTOTIC STABILITY}

In this section we will put some conditions on operators $A$ and $F(0)$ so as to define a Liapunov function and apply Theorem 1.4 to obtain the uniform asymptotic stability.

First, we allow $F(0)=0$. This case is somewhat delicate because the leading matrix in Eq. (1.7) and Eq. (1.9) has zero as an eigenvalue. This problem is largely eliminated by the form of Eq. (1.7) and Eq. (1.9), however.

Theorem 2.1. Suppose that Eq. (1.3) (and Eq. (1.4)) has a unique solution (when $\phi$ satisfies certain conditions), and suppose that for some constant $\alpha>0$,

$$
\langle A x, x\rangle \leq-\alpha\langle x, x\rangle, \quad x \in D(A)
$$

and

$$
\langle F(0) x, x\rangle \geq 0, \quad x \in X .
$$

Suppose also that $\int_{0}^{\infty}\|F(s)\| d s \equiv \Gamma<1$ and $\int_{0}^{\infty}\left\|F^{\prime}(s)\right\| d s \equiv \Delta<\infty$. If

$$
\rho \equiv \alpha-(1-\Gamma)^{-2}\left(\frac{3}{2}\|F(0)\|+6 \sqrt{3} \delta \Delta\right)-\frac{3}{2}\|F(0)\|-\frac{31 \sqrt{3}}{4} \delta \Delta>0,
$$

where $\delta>1$ is a constant, then $z \equiv 0$ of Eqs. (1.7) and (1.9), hence $x \equiv 0$ of Eqs. (1.3) and (1.4), are uniformly asymptotically stable.

Proof. As remarked before, under the above conditions it is known ([3, Theorems 3.5 and 4.3]) that $z \equiv 0$ of Eqs. (1.7) and (1.9) are uniformly stable. So we only need to prove that other conditions in Definition 1.3 are satisfied. Toward this end, we prove that conditions in Theorem 1.4 are satisfied. For $z=(x, w)^{T}$, define

$$
V(z)=\langle x, x\rangle-2\langle x, w\rangle+\frac{3}{2}\langle w, w\rangle
$$

As in [3], we have

$$
\begin{aligned}
V(z) & \geq\|x\|^{2}-2\|x\|\|w\|+\frac{3}{2}\|w\|^{2} \\
& =(\|x\|-\|w\|)^{2}+\frac{1}{2}\|w\|^{2} \\
& =\frac{1}{6}(3\|w\|-2\|x\|)^{2}+\frac{1}{3}\|x\|^{2} .
\end{aligned}
$$

So we obtain

$$
\frac{1}{12}\|z\|^{2} \leq V(z) \leq 3\|z\|^{2} .
$$

Next, let $z(t)=z\left(t, t_{0}, \xi\right)$ be a solution of Eq. (1.7) (or Eq. (1.9)). Differentiating $V(z(t))$ with respect to $t$ yields

$$
\begin{aligned}
V^{\prime}(z(t))= & \langle A w(t), w(t)\rangle-2\langle x(t), F(0) x(t)\rangle-2\left\langle x(t), F^{\prime} * x(t)\right\rangle \\
& +3\langle F(0) x(t), w(t)\rangle+3\left\langle F^{\prime} * x(t), w(t)\right\rangle \\
\leq & -\alpha\|w(t)\|^{2}+3\|F(0)\|\|x(t)\|\|w(t)\| \\
& +\{2\|x(t)\|+3\|w(t)\|\}\left\|F^{\prime} * x(t)\right\|, \quad t \geq t_{0},
\end{aligned}
$$


where

$$
F^{\prime} * x(t)=\int_{\#}^{t} F^{\prime}(t-s) x(s) d s \quad(\#=0 \text { or }-\infty) .
$$

Define $h(t)=\delta^{2} t, t \geq 0$, and define $u_{j}=4 / j$. Note that from [3, Theorem 4.2], one can also check that solutions of Eq. (1.7) and Eq. (1.9) are bounded. That is, for $r \equiv 1$, there is a constant $M>1$ such that for solutions $z\left(t, t_{0}, \xi\right)$ with $\|\xi(s)\| \leq 1$, here $s \in\left[0, t_{0}\right]$ for Eq. (1.7) and $s \leq t_{0}$ for Eq. (1.9), one has $\|z(t)\| \leq M, t \geq t_{0}$.

Next, as $\Delta=\int_{0}^{\infty}\left\|F^{\prime}(s)\right\| d s<\infty$, there is $r_{j}>j$ such that

$$
\int_{r_{j}}^{\infty}\left\|F^{\prime}(s)\right\| d s \leq \rho(1-\Gamma)^{2} / 6 M^{2} j^{2} .
$$

Now, if $V(z(s))<\delta^{2} V(z(t))$ for $t_{0} \leq t-r_{j} \leq s \leq t$, then from (2.5),

$$
\begin{aligned}
\|x(s)\|^{2} & \leq 3 V(z(s)) \leq 3 \delta^{2} V(z(t)), & & t-r_{j} \leq s \leq t, \\
\|w(s)\|^{2} & \leq 2 V(z(s)) \leq 2 \delta^{2} V(z(t)), & & t-r_{j} \leq s \leq t .
\end{aligned}
$$

Thus

$$
\left\|F^{\prime} * x(t)\right\| \leq \int_{\#}^{t}\left\|F^{\prime}(t-s) x(s)\right\| d s=\int_{\#}^{t-r_{j}}+\int_{t-r_{j}}^{t} \equiv I_{1}+I_{2},
$$

with

$$
\begin{gathered}
I_{1} \leq M \int_{r_{j}}^{\infty}\left\|F^{\prime}(s)\right\| d s \leq \rho(1-\Gamma)^{2} / 6 M j^{2}, \\
I_{2} \leq \delta \sqrt{3 V(z(t))} \int_{0}^{\infty}\left\|F^{\prime}(s)\right\| d s=\delta \Delta \sqrt{3 V(z(t))} .
\end{gathered}
$$

Therefore, if $V(z(s))<\delta^{2} V(z(t))$ for $t_{0} \leq t-r_{j} \leq s \leq t$, then (2.7) becomes

$$
\begin{aligned}
V^{\prime}(z(t)) \leq & -\alpha\|w(t)\|^{2}+\frac{3}{2}\|F(0)\|\left\{\|x(t)\|^{2}+\|w(t)\|^{2}\right\} \\
& +\{2\|x(t)\|+3\|w(t)\|\} I_{1}+\{2\|x(t)\|+3\|w(t)\|\} I_{2} \\
\leq & -\alpha\|w(t)\|^{2}+\frac{3}{2}\|F(0)\|\left\{\|x(t)\|^{2}+\|w(t)\|^{2}\right\} \\
& +\{2\|x(t)\|+3\|w(t)\|\} \delta \Delta \sqrt{3 V(z(t))} \\
& +\{2\|x(t)\|+3\|w(t)\|\} I_{1} \\
\leq & -\alpha\|w(t)\|^{2}+\frac{3}{2}\|F(0)\|\left\{\|x(t)\|^{2}+\|w(t)\|^{2}\right\} \\
& +\sqrt{3} \delta \Delta\left\{\|x(t)\|^{2}+V(z(t))\right\}+\frac{3 \sqrt{3}}{2} \delta \Delta\left\{\|w(t)\|^{2}+V(z(t))\right\} \\
& +\{2\|x(t)\|+3\|w(t)\|\} I_{1} \\
= & \|x(t)\|^{2}\left\{\frac{3}{2}\|F(0)\|+\sqrt{3} \delta \Delta\right\}-\|w(t)\|^{2}\left\{\alpha-\frac{3}{2}\|F(0)\|-\frac{3 \sqrt{3}}{2} \delta \Delta\right\} \\
& +\frac{5}{2} \sqrt{3} \delta \Delta V(z(t))+\{2\|x(t)\|+3\|w(t)\|\} I_{1} \\
\leq & \|x(t)\|^{2}\left\{\frac{3}{2}\|F(0)\|+\sqrt{3} \delta \Delta\right\}-\|w(t)\|^{2}\left\{\alpha-\frac{3}{2}\|F(0)\|-\frac{3 \sqrt{3}}{2} \delta \Delta\right\} \\
& +\frac{5}{2} \sqrt{3} \delta \Delta\left\{2\|x(t)\|^{2}+\frac{5}{2}\|w(t)\|^{2}\right\}+\{2\|x(t)\|+3\|w(t)\|\} I_{1} \\
= & \|x(t)\|^{2}\left\{\frac{3}{2}\|F(0)\|+6 \sqrt{3} \delta \Delta\right\}-\|w(t)\|^{2}\left\{\alpha-\frac{3}{2}\|F(0)\|-\frac{31 \sqrt{3}}{4} \delta \Delta\right\} \\
& +\{2\|x(t)\|+3\|w(t)\|\} I_{1} \\
\leq & \|x(t)\|^{2}\left\{\frac{3}{2}\|F(0)\|+6 \sqrt{3} \delta \Delta\right\}-\|w(t)\|^{2}\left\{\alpha-\frac{3}{2}\|F(0)\|-\frac{31 \sqrt{3}}{4} \delta \Delta\right\} \\
& +\rho(1-\Gamma)^{2} / 2 j^{2} .
\end{aligned}
$$


Next, it is proven in [3] that

So we obtain

$$
\|x(t)\| \leq(1-\Gamma)^{-1}\|w(t)\|
$$

$$
\begin{aligned}
V^{\prime}(z(t)) & \leq-\rho\|w(t)\|^{2}+\rho(1-\Gamma)^{2} / 2 j^{2} \\
& =-\frac{\rho}{2}\|w(t)\|^{2}-\frac{\rho}{2}\|w(t)\|^{2}+\rho(1-\Gamma)^{2} / 2 j^{2} \\
& \leq-\frac{\rho}{2}\|w(t)\|^{2}-\frac{\rho(1-\Gamma)^{2}}{2}\|x(t)\|^{2}+\rho(1-\Gamma)^{2} / 2 j^{2} \\
& \leq-\frac{\rho(1-\Gamma)^{2}}{2}\left\{\|x(t)\|^{2}+\|w(t)\|^{2}\right\}+\rho(1-\Gamma)^{2} / 2 j^{2} \\
& \leq-\frac{\rho(1-\Gamma)^{2}}{4}\|z(t)\|^{2}+\rho(1-\Gamma)^{2} / 2 j^{2} \\
& \equiv-w_{j}(\|z(t)\|) .
\end{aligned}
$$

Also note that for $t \geq r_{j}+t_{0}$ and $\|z(t)\| \geq u_{j} / 2=2 / j$, we have

$$
\begin{aligned}
w_{j}(\|z(t)\|) & \geq \rho(1-\Gamma)^{2} / j^{2}-\rho(1-\Gamma)^{2} / 2 j^{2} \\
& =\rho(1-\Gamma)^{2} / 2 j^{2}>0 .
\end{aligned}
$$

Therefore, conditions in Theorem 1.4 are satisfied. Hence, for $r \equiv 1$ and $M>1$ from the boundedness ( $M$ is fixed then) and any $\varepsilon>0$ there exists $T=T(\varepsilon, M)=T(\varepsilon)>0$ such that when $\|\xi(s)\|<r=1$ (here $s \in\left[0, t_{0}\right]$ for Eq. (1.7) and $s \leq t_{0}$ for Eq. (1.9)), which implies $\|z(t)\|=\left\|z\left(t, t_{0}, \xi\right)\right\| \leq M$ for all $t$, one has $\|z(t)\|<\varepsilon$ for $t \geq T+t_{0}$. Thus the desired result follows.

In case $\langle F(0) x, x\rangle \geq \beta\langle x, x\rangle, \beta>0$, we are able to obtain, using basically the same proof as above ([3, Theorem 3.4]), a stronger result without assuming $F \in L^{1}(0, \infty)$. We omit the details here.

Theorem 2.2. Suppose that Eq. (1.3) (and Eq. (1.4)) has a unique solution (when $\phi$ satisfies certain conditions), and suppose that for some constants $\alpha>0$ and $\beta>0$,

$$
\langle A x, x\rangle \leq-\alpha\langle x, x\rangle, \quad 2 \in D(A)
$$

and

$$
\langle F(0) x, x\rangle \geq \beta\langle x, x\rangle, \quad x \in X .
$$

Suppose also that $\int_{0}^{\infty}\left\|F^{\prime}(s)\right\| d s \equiv \Delta<\infty$. If

$$
\rho \equiv \min \left\{\alpha-\frac{31 \sqrt{3}}{4} \delta \Delta, 2 \beta-6 \sqrt{3} \delta \Delta\right\}-\frac{3}{2}\|F(0)\|>0,
$$

where $\delta>1$ is a constant, then $z \equiv 0$ of Eqs. (1.7) and (1.9), hence $x \equiv 0$ of Eqs. (1.3) and (1.4), are uniformly asymptotically stable.

Remark 2.3. It is known that $A \equiv \frac{\partial^{2}}{\partial x^{2}}$ with domain $H_{0}^{1}(0,1) \cap H^{2}(0,1)$ satisfy (2.1) on $X=L^{2}(0,1)$ with $\alpha=1$. Thus applications can be carried out. We omit them here for simplicity.

\section{ACKNOWLEDGMENT}

The author would like to thank the referees for their valuable suggestions and comments. 


\section{REFERENCES}

1. T. Burton, Stability and periodic solutions of ordinary differential equations and functional differential equations, Academic Press, New York, 1985.

2. R. Fabiano and K. Ito, Semigroup theory and numerical approximation for equations in linear viscoelasticity, SIAM J. Math. Anal. 21 (1990), 374-393.

3. R. Grimmer and J. Liu, Liapunov-Razumikhin methods for integrodifferential equations in Hilbert space, Delay and Differential Equations (A. Fink, R. Miller, and W. Kliemann, eds.), World Scientific, London, 1992, pp. 9-24.

4. C. Gripenberg, S.-O. Londen, and O. Staffans, Volterra integral and functional equations, Cambridge Univ. Press, Cambridge, 1990, pp. 12-13.

5. R. Grimmer and G. Seifert, Stability propeties of Volterra integrodifferential equations, J. Differential Equations 19 (1975), 142-166.

6. G. Seifert, Liapunov-Razumikhin conditions for stability and boundedness of functional differential equations of Volterra type, J. Differential Equations 14 (1973), 424-430.

7. _ Liapunov-Razumikhin conditions for asymptotic stability in functional differential equations of Volterra type, J. Differential Equations 16 (1974), 289-297.

Department of Mathematics, James Madison University, Harrisonburg, Virginia 22807

E-mail address: Liu@math.jmu.edu 